\title{
Synergistic antitumor effect of brusatol combined with cisplatin on colorectal cancer cells
}

\author{
HAI-MING CHEN ${ }^{1-3^{*}}$, ZHENG-QUAN LAI $^{4 *}$, HUI-JUN LIAO $^{5}$, JIAN-HUI XIE $^{1}$, YAN-FANG XIAN ${ }^{4}$, \\ YUN-LONG CHEN ${ }^{4,8}$, SIU-PO IP ${ }^{4}$, ZHI-XIU LIN ${ }^{4}$ and ZI-REN SU ${ }^{2,6,7}$ \\ ${ }^{1}$ The Second Affiliated Hospital; ${ }^{2}$ College of Chinese Medicine; ${ }^{3}$ Postdoctoral Programme, \\ Guangzhou University of Chinese Medicine, Guangzhou, Guangdong 510006; ${ }^{4}$ School of Chinese Medicine, \\ Faculty of Medicine, The Chinese University of Hong Kong, Hong Kong, SAR 999077; ${ }^{5}$ Department of Clinical Pharmacy \\ and Pharmaceutical Services, Shenzhen Sixth People's Hospital (Nanshan Hospital), Shenzhen 518060; \\ ${ }^{6}$ Dongguan Mathematical Engineering Academy of Chinese Medicine, Guangzhou University of Chinese Medicine, \\ Dongguan, Guangdong 523808; ${ }^{7}$ Guangdong Provincial Key Laboratory of New Chinese Medicinal Development and Research, \\ Guangzhou University of Chinese Medicine, Guangzhou, Guangdong 510006; ${ }^{8}$ School of Chinese Materia Medica, \\ Tianjin University of Traditional Chinese Medicine, Tianjin 300193, P.R. China
}

Received June 23, 2016; Accepted December 21, 2017

DOI: $10.3892 /$ ijmm.2018.3372

\begin{abstract}
Colorectal cancer (CRC) is a common and life-threatening type of malignant cancer, which is associated with a high mortality rate. Cisplatin (CDDP) is a commonly used chemotherapy drug with significant side effects. Brusatol (BR) is one of the principal chemical compounds isolated from the Chinese herb Bruceae Fructus, which has been reported to markedly inhibit the proliferation of numerous cancer cell lines. The present study aimed to investigate the possible synergistic anticancer effects of CDDP combined with BR on CT-26 cells, and to evaluate the underlying mechanisms of action. The growth inhibitory effects of BR, CDDP, and BR and CDDP cotreatment on CT-26 cells were assessed by MTT assay. Cell apoptosis were determined by flow cytometry and western blot analysis. The results indicated that compared with single-agent treatment, cotreatment of CT-26 cells with
\end{abstract}

Correspondence to: Professor Zi-Ren Su, College of Chinese Medicine, Guangzhou University of Chinese Medicine, 232 Waihuan Dong Road, Guangzhou Higher Education Mega Center, Guangzhou, Guangdong 510006, P.R. China

E-mail: suziren@126.com

Professor Zhi-Xiu Lin, School of Chinese Medicine, Faculty of Medicine, The Chinese University of Hong Kong, Shatin, N.T., Hong Kong, SAR 999077, P.R. China

E-mail: linzx@cuhk.edu.hk

*Contributed equally

Abbreviations: $\mathrm{BR}$, brusatol; $\mathrm{CDDP}$, cisplatin; $\mathrm{CRC}$, colorectal cancer; PI, propidium iodide; PS, phosphatidylserine; TBS-T, Tris-buffered saline containing $0.1 \%$ Tween-20

Key words: brusatol, cisplatin, synergistic effect, apoptosis, CT-26
CDDP and BR synergistically inhibited cell proliferation and increased cellular apoptosis. Furthermore, treatment of CT-26 cells with CDDP and BR resulted in a marked increase in the release of cytosolic cytochrome $c$, decreased expression of procaspase- 3 and procaspase-9, and upregulation of the B-cell lymphoma 2 (Bcl-2)-associated X protein/Bcl-2 ratio compared with treatment with BR or CDDP alone. These results strongly suggested that the combination of CDDP and BR was able to produce a synergistic antitumor effect in CRC cells, thus providing a solid foundation for further development of this combination regimen into an effective therapeutic method for CRC.

\section{Introduction}

Colorectal cancer (CRC) is one of the most common types of cancer in the Western world, and is the second most common cause of cancer-associated mortality in the world (1-3). It is estimated that $>1.3$ million people worldwide are affected by CRC annually (4). The worldwide threat posed by CRC is increasing, which is largely due to an aging population and the increased adoption of Westernized diets in developed and developing countries (5-7).

At present, surgery, chemotherapy and radiotherapy are applied as the main therapeutic approaches for the treatment of CRC in clinical practice (8-10). Among them, systemic chemotherapy is regarded as a promising therapeutic approach, due to its ability to elicit a good therapeutic response, improve quality of life and prolong survival (9). Cisplatin (CDDP) is one of the most frequently used chemotherapy drugs, which exerts a strong therapeutic effect; however, some tumor types, including colon, ovarian and lung cancer, have not exhibited satisfactory results in response to CDDP (11). Therefore, the enhancement of efficacy by specific compounds may provide a valuable contribution to the treatment of cancer based on CDDP chemotherapy. For this purpose, the development of 
combined application of CDDP with other safe and effective agents has been the focus of research.

Currently, herbal medicines or natural compounds, either used as a monotherapy or combined with conventional chemotherapeutic agents, have been reported to exert beneficial effects on the treatment of various types of cancer (12). Bruceae Fructus refers to the fruit of Brucea javanica (L.) Merr. ('Ya-Dan-Zi' in Chinese), and was initially recorded in Supplementations to the Compendium of Chinese Materia Medica. Bruceae Fructus has been applied to treat various ailments, including cancer, amoebic dysentery and malaria, since the Ming Dynasty (1364-1644 AD) $(13,14)$. The antitumor activity of Bruceae Fructus is regarded as one of the most important biological activities of this plant, and it has been commonly prescribed to treat various types of cancer in China. In previous years, emerging evidence has been provided with regards to the antitumor activity of Bruceae Fructus (13).

$B$. javanica is rich in quassinoids, which are considered the predominant ingredients responsible for its marked antitumor activity (15). Brusatol (BR; $\mathrm{C}_{26} \mathrm{H}_{32} \mathrm{O}_{11}$ ), the chemical structure of which is presented in Fig. 1, is one of the major quassinoids isolated from $B$. javanica. This compound has been reported to exert marked anti-inflammatory (16), antimalarial (17) and antitumor activities (18-21). In addition, BR has been demonstrated to uniquely block the nuclear factor erythroid 2-related factor 2 pathway, thus sensitizing various cancer cells in vitro and A549 mouse xenografts to chemotherapeutic agents, including CDDP. These findings suggested that BR may be considered a promising candidate for combating chemoresistance and for further development into an effective adjuvant for chemotherapy drugs (22). However, whether CDDP combined with BR exerts synergistic antitumor activity on CT-26 CRC cells remains unclear. Therefore, the present study aimed to investigate the possible effects of BR alone, and in combination with CDDP, on CT-26 CRC cells, and to evaluate the potential mechanism.

\section{Materials and methods}

Reagents and chemicals. BR (CAS: 14907-98-3; PubChem CID: 73432) was isolated from Bruceae Fructus in our laboratory. Briefly, the seeds of B. javanica were extracted twice with $95 \% \mathrm{EtOH}$ for $2 \mathrm{~h}$, concentrated to give a crude extract and suspended in $\mathrm{H}_{2} \mathrm{O}$. The aqueous layer was further extracted with EtOAc and evaporated under vacuum to afford extracts and subjected to silica gel column chromatography eluted with a gradient of $\mathrm{CH}_{2} \mathrm{Cl}_{2}-\mathrm{MeOH}$ (100:0-100:20). The $\mathrm{CH}_{2} \mathrm{Cl}_{2}-$ $\mathrm{MeOH}(100: 1)$ eluate was evaporated to yield a residue, which was further purified by repeated recrystallization to obtain a white powder. The chemical structure of BR was confirmed and purity was determined to be $>98 \%$ (21). CDDP and MTT were obtained from Sigma-Aldrich; Merck KGaA (Darmstadt, Germany). Antibodies against caspase-3 (sc-113427) and caspase-9 (sc-56073), cytochrome $c$ (sc-13156), B-cell lymphoma 2 (Bcl-2)-associated X protein (Bax; sc-20067), Bcl-2 (sc-509) and $\beta$-actin (sc-47778) were obtained from Santa Cruz Biotechnology, Inc. (Dallas, TX, USA). All other chemicals and reagents were of analytical grade.

Cell culture. The murine CT-26 CRC cell line was purchased from the American Type Culture Collection (Manassas,

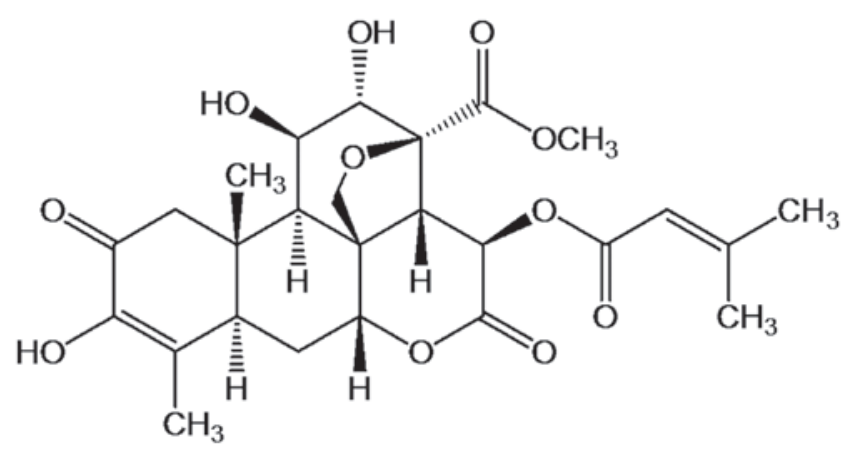

Figure 1. Chemical structure of brusatol.

VA, USA). CT-26 cells were routinely grown in RPMI-1640 medium supplemented with $10 \%$ fetal bovine serum (both from Gibco; Thermo Fisher Scientific, Inc., Waltham, MA, USA) and $1 \%$ penicillin-streptomycin at $37^{\circ} \mathrm{C}$ in a humidified atmosphere containing $5 \% \mathrm{CO}_{2}$.

In vitro cytotoxicity assays. In vitro $\mathrm{BR}$ and $\mathrm{CDDP}$ cytotoxic effects against the CRC cell line were measured using an MTT assay. Briefly, CT-26 cells in logarithmic growth were seeded onto a 96-well plate at a density of $4 \times 10^{3}$ cells/well. After $24 \mathrm{~h}$ of incubation at $37^{\circ} \mathrm{C}$, fresh medium containing a series of concentrations of BR $(0.05,0.15,0.45,1.35,4.05$ and $12.15 \mu \mathrm{g} / \mathrm{ml})$ and CDDP $(0.05,0.15,0.45,1.35,4.05$ and $12.15 \mu \mathrm{g} / \mathrm{ml})$ was added at $100 \mu \mathrm{l} /$ well; each concentration was used to treat six replicate wells. After $48 \mathrm{~h}$ of incubation at $37^{\circ} \mathrm{C}$, the cells were further incubated with MTT $(10 \mathrm{mg} / \mathrm{ml})$ at $37^{\circ} \mathrm{C}$ for $4 \mathrm{~h}$. The supernatant was then removed and the precipitate was dissolved with $100 \mu \mathrm{l}$ dimethyl sulfoxide. Absorbance was measured using a microplate reader (EXL808; BioTek Instruments, Inc., Winooski, VT, USA) at a wavelength of $490 \mathrm{~nm}$. Cytotoxicity was expressed as the concentration of BR and CDDP that inhibited cell growth by $50 \%$ [half maximal inhibitory concentration $\left(\mathrm{IC}_{50}\right)$ value]. The inhibitory rate was calculated according to the following formula: Inhibitory rate $(\%)=\left(1-\mathrm{OD}_{\text {experiment group }} / \mathrm{OD}_{\text {control group }}\right) \times 100 \%$; where OD refers to optical density. The possible synergistic effect of BR combined with CDDP was investigated by exposing CT-26 cells to various concentrations of each agent alone or in combination for $48 \mathrm{~h}$. The synergistic effect was assessed using CalcuSyn software 2.0 (Biosoft, Cambridge, UK), which determines the combination index $(\mathrm{CI})$ based on that described by $\mathrm{Chou}$ and Talalay $(23,24) . \mathrm{CI}=1, \mathrm{CI}<1$ and $\mathrm{CI}>1$ represented an additive effect, synergism and antagonism, respectively (23).

Morphological observation of nuclear alterations. CT-26 cancer cells were grown on coverslips placed in 6-well plates and were treated with a single drug (BR or CDDP) or combination for $48 \mathrm{~h}$ (incubation at $37^{\circ} \mathrm{C}$ ). After washing twice, Hoechst 33342 (Hoechst staining kit; Beyotime Institute of Biotechnology, Beijing, China) was used to stain the cells for $1 \mathrm{~h}$ at room temperature. Subsequently, cell morphology was observed and images were captured from random visual fields using a fluorescence microscopy (Zeiss GmbH, Jena, Germany).

Flowcytometricanalysisofapoptosis.The Annexin V-fluorescein isothiocyanate (FITC) kit (Thermo Fisher Scientific, Inc.) was 


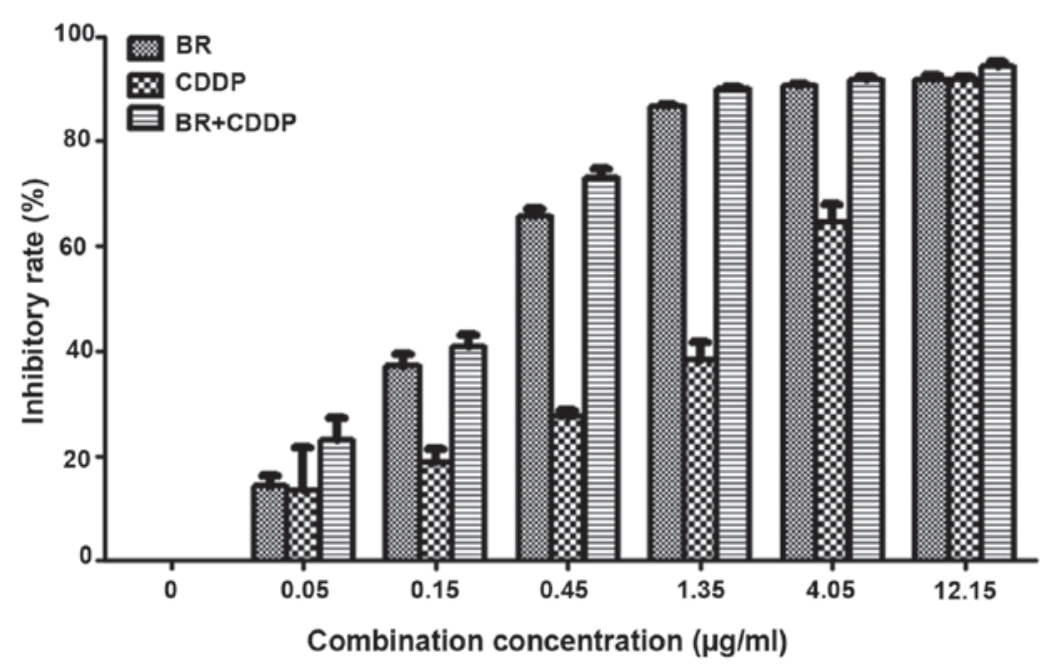

Figure 2. Inhibitory rate of CT-26 cells following treatment with various concentrations of BR $(0.75-15 \mu \mathrm{g} / \mathrm{ml})$ and CDDP $(0.75-15 \mu \mathrm{g} / \mathrm{ml})$ either alone or in combination. Data are presented as the means \pm standard deviation of three independent experiments. BR, brusatol; CDDP, cisplatin.

Table I. $\mathrm{IC}_{50}$ values of BR and CDDP either alone or in combination on CT-26 cells.

\begin{tabular}{lc}
\hline Agent & $\mathrm{IC}_{50}$ value $(\mu \mathrm{g} / \mathrm{ml})$ \\
\hline BR & $0.27 \pm 0.01$ \\
CDDP & $1.44 \pm 0.22$ \\
BR + CDDP & $0.19 \pm 0.02$ \\
\hline
\end{tabular}

BR, brusatol; CDDP, cisplatin; IC50, half maximal inhibitory concentration.

used to determine cellular apoptosis. After exposure to BR $(0.27 \mu \mathrm{g} / \mathrm{ml}), \operatorname{CDDP}(1.44 \mu \mathrm{g} / \mathrm{ml})$, or their combination for $48 \mathrm{~h}$ in $37^{\circ} \mathrm{C}, \mathrm{CT}-26$ cells were collected, washed twice with PBS and subjected to centrifugation at $180 \mathrm{xg}$ for $5 \mathrm{~min}$ at room temperature. Subsequently, the cell pellet was resuspended and treated with Annexin V-FITC and propidium iodide (PI) solutions. After incubating for $15 \mathrm{~min}$ at room temperature in the dark, additional Annexin $\mathrm{V}$ binding buffer $(400 \mu \mathrm{l})$ was added to each tube and the cells were analyzed using a Cytomics ${ }^{\mathrm{TM}}$ FC500 flow cytometer (Beckman Coulter, Inc., Brea, CA, USA) and disposed with FlowJo 7.6.5. (FlowJo, LLC, Ashland, OR, USA).

Western blot analysis. After treatment, the cells were harvested and lysed with radioimmunoprecipitation assay buffer (Cell Signaling Technology, Inc., Boston, MA, USA) supplemented with cocktail (Roche, Penzberg, Germany). Protein concentration was determined using a BCA Protein Assay kit (cat. no. 23225, Thermo Fisher Scientific, Inc.) and about $40 \mu \mathrm{g}$ protein were separated with $10 \%$ SDS-PAGE by electrophoresis and were transferred to polyvinylidene fluoride (PVDF) membranes (Immobilon; EMD Millipore, Billerica, MA, USA) using trans-blotting apparatus (Bio-Rad Laboratories, Inc., Hercules, CA, USA). Non-fat milk (5\%, w/v) dissolved in Tris-buffered saline containing $0.1 \%$ Tween-20 (TBS-T) was used to block the PVDF membranes. The membranes were then incubated with the following primary antibodies:
Procaspase-3 (1:200), procaspase-9 (1:200), cytochrome $c$ (1:200), Bcl-2 (1:200), Bax (1:200) and $\beta$-actin (Santa Cruz Biotechnology, Inc.) overnight at $4^{\circ} \mathrm{C}$. After washing with TBS-T three times, the membranes were incubated with the appropriate secondary antibodies (1:1,000; sc-2350, sc-2005 and sc-2370; Santa Cruz Biotechnology, Inc.). Finally, the protein bands were developed using enhanced chemiluminescence western blot detection reagents (GE Healthcare, Chicago, IL, USA) and were analyzed using ImageJ software $1.51 \mathrm{~s}$ (National Institutes of Health, Bethesda, MD, USA).

Statistical analysis. SPSS 19.0 (IBM Corp., Armonk, NY, USA) was used to conduct all statistical analyses. Data are presented as the means \pm standard deviation. One-way analysis of variance was used for multiple group comparisons, followed by Dunnett's test to detect intergroup differences. $\mathrm{P}<0.05$ was considered to indicate a statistically significant difference.

\section{Results}

Synergistic cytotoxic effects of BR in combination with CDDP on CT-26 CRC cells. To explore the possible synergistic cytotoxicity of BR in combination with CDDP, the present study investigated the effects of BR and CDDP cotreatment on CT-26 cell viability using an MTT assay. CT-26 cells were treated with various concentrations of $\mathrm{BR}(0.05,0.15,0.45$, $1.35,4.05$ and $12.15 \mu \mathrm{g} / \mathrm{ml})$ and CDDP $(0.05,0.15,0.45,1.35$, 4.05 and $12.15 \mu \mathrm{g} / \mathrm{ml}$ ) for $48 \mathrm{~h}$, either alone or in combination.

The inhibitory effects on the proliferation of CT-26 cells and $\mathrm{IC}_{50}$ values are presented in Fig. 2 and Table I, respectively. Following treatment with BR and CDDP for $48 \mathrm{~h}$, the viability of CT-26 cells was reduced in a dose-dependent manner, with $\mathrm{IC}_{50}$ values of $0.27 \pm 0.01$ and $1.44 \pm 0.22 \mu \mathrm{g} / \mathrm{ml}$, respectively. When BR was combined with CDDP at a constant concentration ratio of 1:1, cell growth inhibition was markedly enhanced compared with single-agent treatment; the $\mathrm{IC}_{50}$ value of $\mathrm{BR}$ and CDDP cotreatment was $0.19 \pm 0.02 \mu \mathrm{g} / \mathrm{ml}$.

The effects of CDDP and BR cotreatment on cell proliferation were revealed to be synergistic, as determined by calculating the CI values (Fig. 3). Isobologram analysis indicated that the 


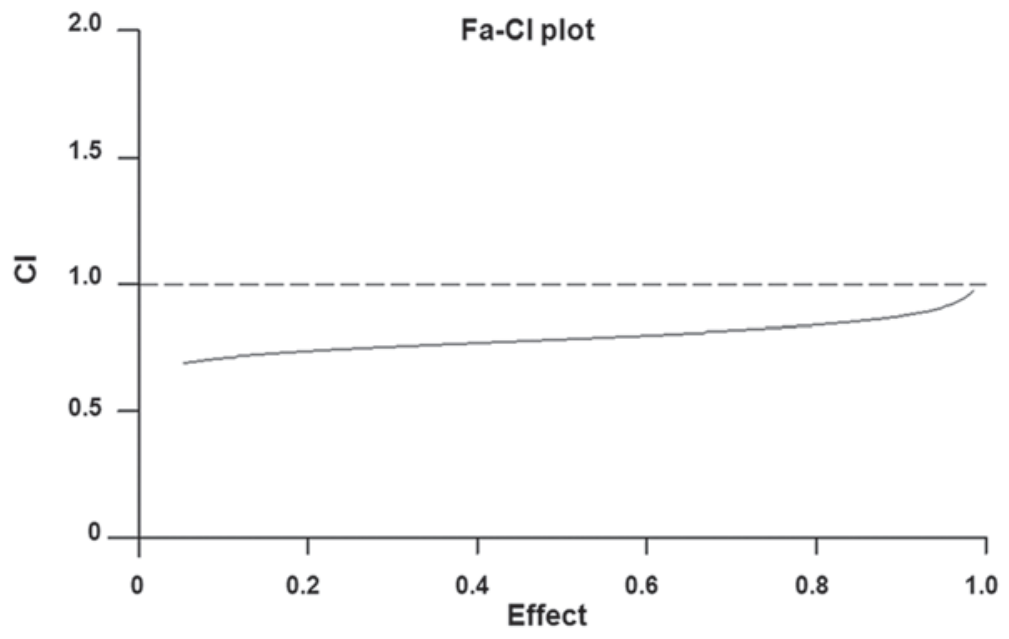

Figure 3. Fa-CI plot for brusatol and cisplatin cotreatment on CT-26 cells. CI, combination index.
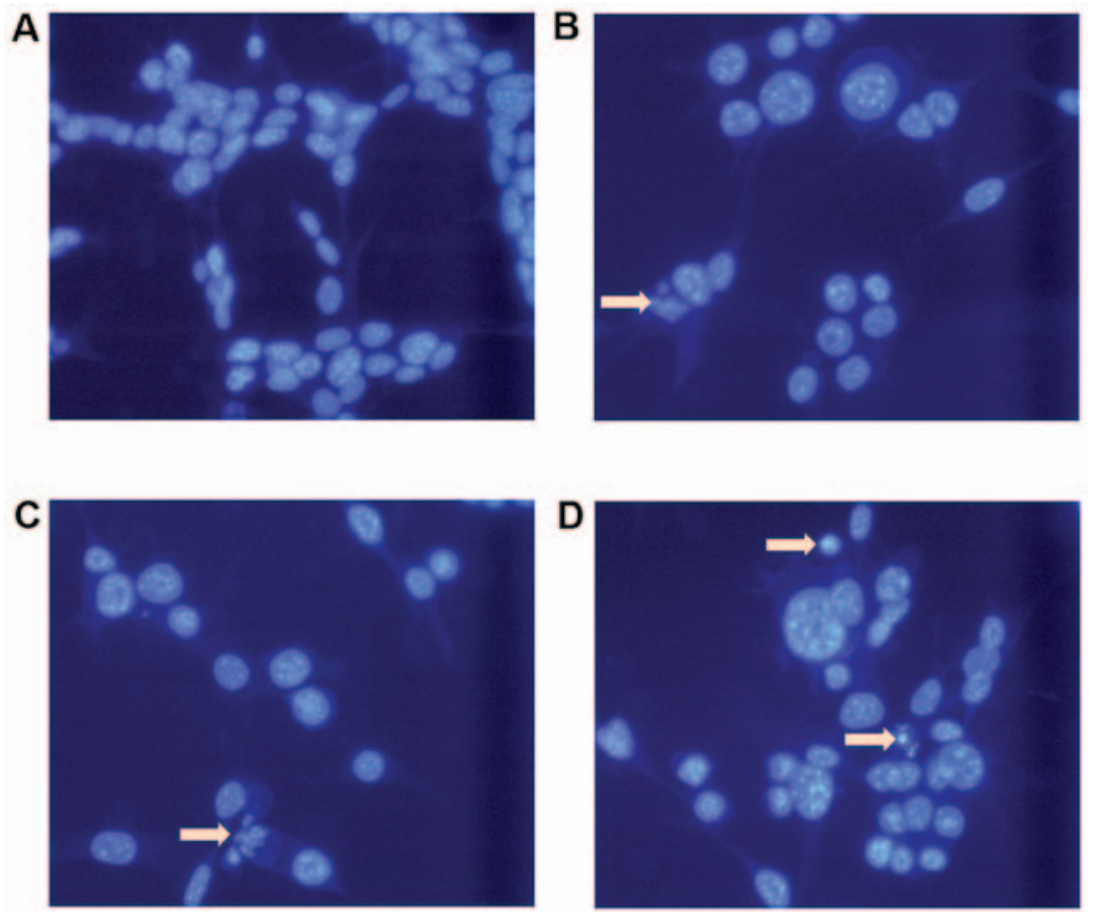

Figure 4. Cellular apoptosis observed with Hoechst 33342 staining (magnification, x200). CT-26 cells were treated with BR $(0.27 \mu \mathrm{g} / \mathrm{ml}), \mathrm{CDDP}(1.44 \mu \mathrm{g} / \mathrm{ml})$, or their combination for $48 \mathrm{~h}$. Chromatin condensation, nuclear fragmentation and apoptotic bodies are indicated by small arrows. (A) Control group; (B) BR $(0.27 \mu \mathrm{g} / \mathrm{ml})$-treated group; (C) CDDP $(1.44 \mu \mathrm{g} / \mathrm{ml})$-treated group; and (D) BR $(0.27 \mu \mathrm{g} / \mathrm{ml})+\operatorname{CDDP}(1.44 \mu \mathrm{g} / \mathrm{ml})$-treated group. BR, brusatol; CDDP, cisplatin

CI value was $<1$, thus suggesting that there was a synergistic effect of BR in combination with CDDP on CT-26 cell inhibition.

Morphological alterations in CT-26 cells. To investigate whether cellular apoptosis was involved in the cytotoxic effects of BR and CDDP cotreatment on CT-26 CRC cells, morphological alterations were observed using Hoechst 33342 nuclear staining (Fig. 4). Compared with the control cells, cells treated with BR and CDDP underwent chromatin condensation, and nuclear fragmentation and shrinkage, which are characteristics of apoptosis. Compared with in the BR and CDDP single treatment groups, cells treated with a combination of BR and CDDP exhibited a more obvious increase in the levels of apoptotic chromatin condensation and the number of dead cells.
Synergistic induction of apoptosis of CT-26 cells by BR and $C D D P$. To further confirm whether the antitumor effects of BR and CDDP cotreatment on CT-26 cells were associated with the induction of apoptosis, Annexin V/PI double staining was used to detect apoptosis of CT-26 cells, which were treated with BR, CDDP and their combination. The proportions of early and late apoptotic cells were quantified using flow cytometric analysis, after labeling cells with PI and Annexin V. As shown in Fig. 5, there was a marked increase in the number of apoptotic cells when CT-26 cells were treated with BR or CDDP. The results indicated that BR and CDDP, either individually or in combination, were able to generate a significant increase in the apoptotic population of CT-26 cells $(\mathrm{P}<0.01$; Fig. 5B). Compared with the BR or CDDP groups, a 
A
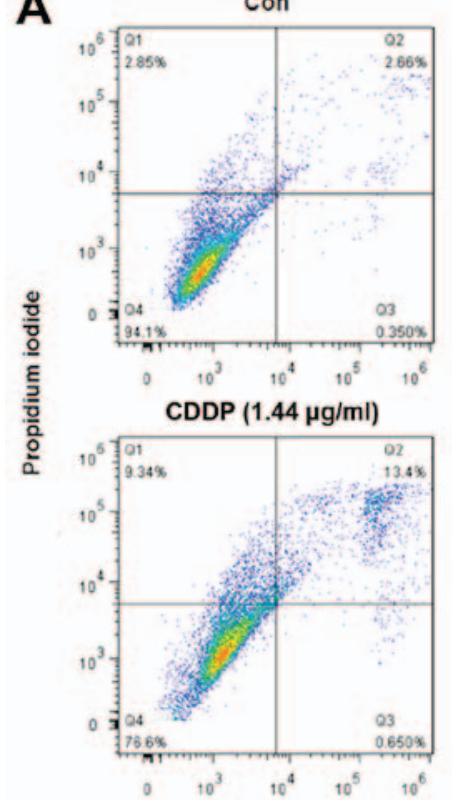

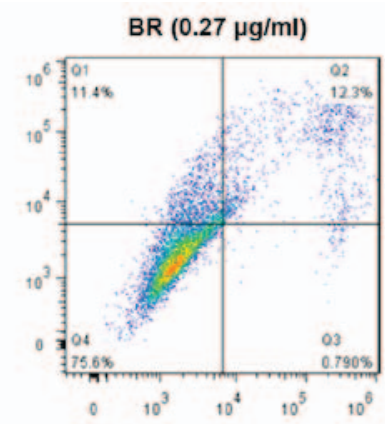

$\operatorname{BR}(0.27 \mu \mathrm{g} / \mathrm{ml})+\operatorname{CDDP}(1.44 \mu \mathrm{g} / \mathrm{ml})$

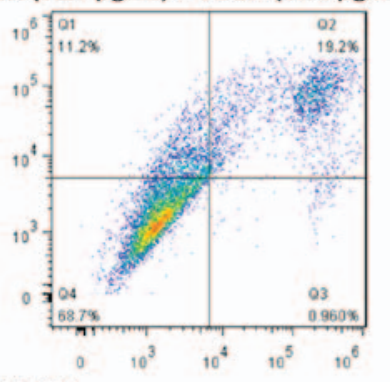

B

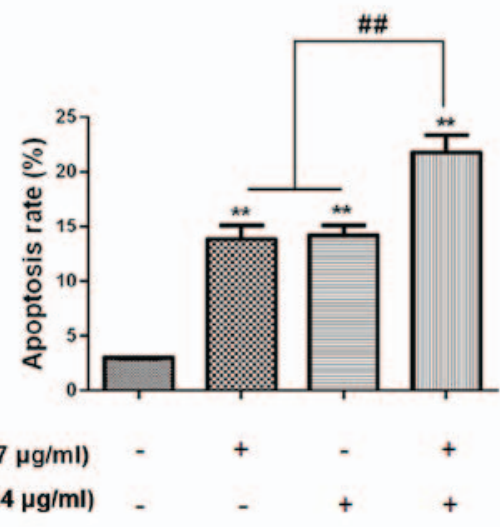

Figure 5. Apoptosis of CT-26 cells mediated by BR and CDDP, alone or in combination. (A) Apoptosis was measured by flow cytometry after $\mathrm{PI} /$ Annexin V-FITC staining. Q1, $\mathrm{PI}^{+}$(cells undergoing necrosis); Q2, Annexin V-FITC ${ }^{+} \mathrm{PI}^{+}$(cells in the late period of apoptosis and undergoing secondary necrosis); Q3, Annexin V-FITC ${ }^{+}$PI $^{-}$(cells in the early period of apoptosis); Q4, Annexin V-FITC- PI $^{-}$(living cells). Total apoptotic rate was calculated as Q2+ Q3. (B) Apototic rates were calculated. The proportion of early and late apoptotic cells stained with Annexin V and PI is presented for each group. Data are presented as the means \pm standard deviation of three independent experiments. ${ }^{* *} \mathrm{P}<0.01$ compared with the Con group; ${ }^{\# \#} \mathrm{P}<0.01$ compared with the BR and CDDP monotherapy groups. BR, brusatol; CDDP, cisplatin; Con, control; FITC, fluorescein isothiocyanate; PI, propidium iodide

A

Procaspase-3

Procaspase-9

$\beta$-actin

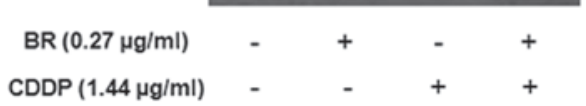

C

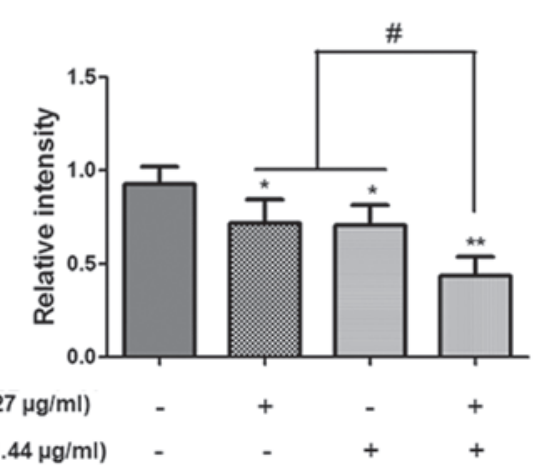

B

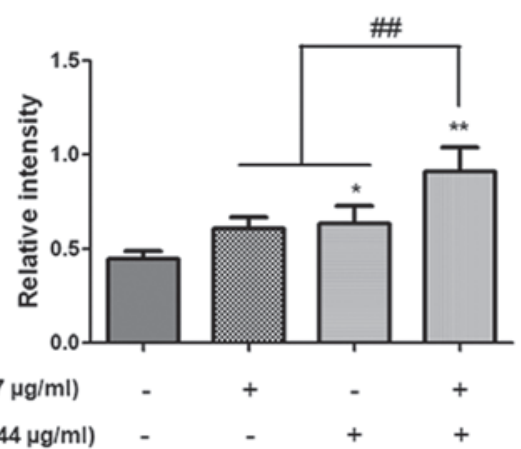

D

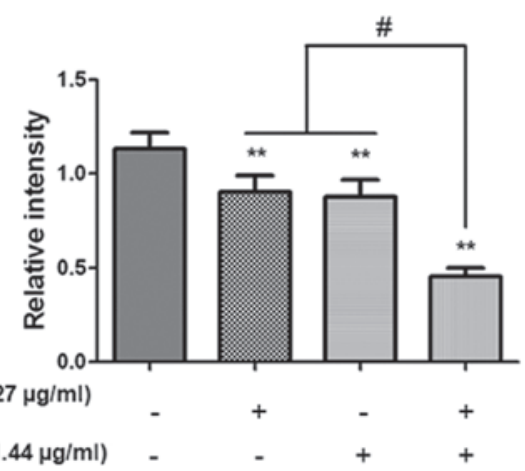

Figure 6. Protein expression levels of procaspase-3, procaspase-9 and cyt $c$ in CT-26 cells treated with BR and CDDP, alone or in combination. (A) Total cell extracts were prepared and subjected to western blot analysis to monitor the protein expression levels of procaspase-3, procaspase-9 and cyt $c$ in CT-26 cells $\beta$-actin was used as the protein loading control. (B-D) Protein expression levels (relative to $\beta$-actin) of (B) cyt $c$, (C) procaspase-3 and (D) procaspase-9 were determined. All data are presented as the the means \pm standard deviation of at least three independent experiments. ${ }^{*} \mathrm{P}<0.05$ and ${ }^{* *} \mathrm{P}<0.01$ compared with the control group; ${ }^{\#} \mathrm{P}<0.05$ and ${ }^{\# \#} \mathrm{P}<0.01$ compared with the BR or CDDP monotherapy groups. BR, brusatol; CDDP, cisplatin; cyt $c$, cytochrome $c$.

significantly greater apoptotic rate was observed in the BR and CDDP cotreatment group $(\mathrm{P}<0.01$; Fig. 5B).
Effects of BR and CDDP on the expression levels of apoptosis-associated proteins in CT-26 cells. According to the 
A

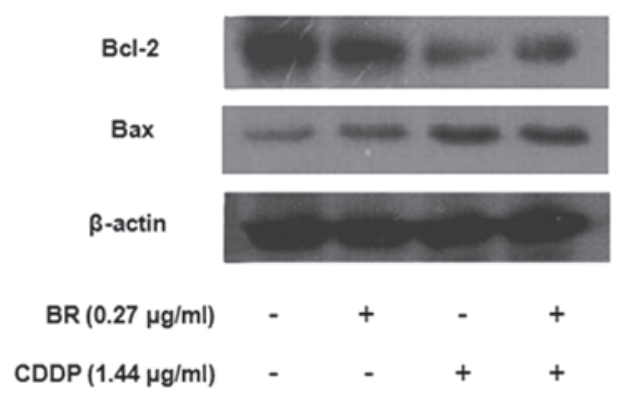

C

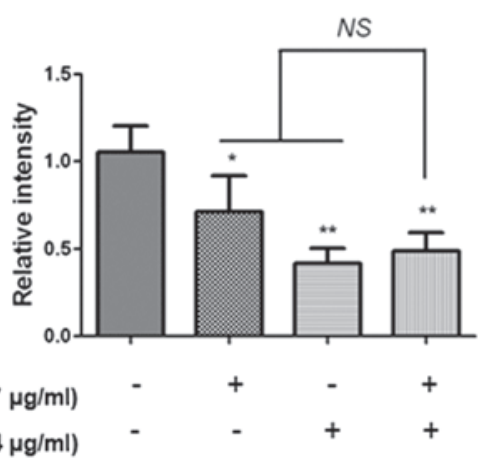

B

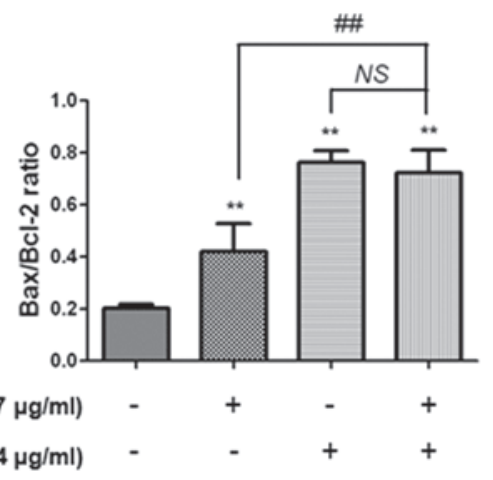

D

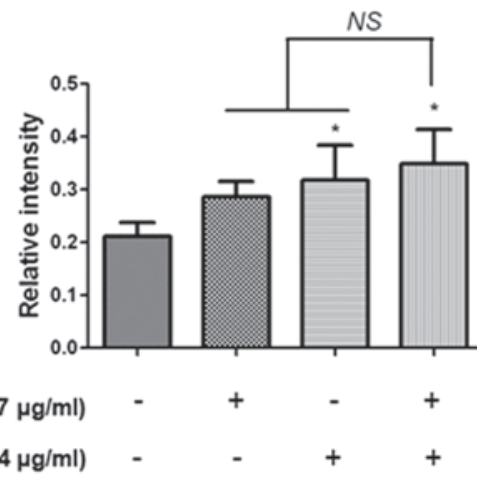

Figure 7. Protein expression levels of Bax, Bcl-2 and Bax/Bcl-2 ratio in CT-26 cells treated with BR and CDDP, alone or in combination. (A) Total cell extracts were prepared and subjected to western blot analysis to monitor the protein expression levels of Bax and Bcl-2 in CT-26 cells. $\beta$-actin was used as the protein loading control. (B) Bax/Bcl-2 ratio, and protein expression levels (relative to $\beta$-actin) of (C) Bcl-2 and (D) Bax were determined. All data are presented as the means \pm standard deviation of at least three independent experiments. ${ }^{~} \mathrm{P}<0.05$ and ${ }^{* *} \mathrm{P}<0.01$ compared with the control group; ${ }^{\# \#} \mathrm{P}<0.01$ compared with the $\mathrm{BR}$ or CDDP monotherapy groups; NS, no statistical significance, $\mathrm{P}>0.05$. Bax, Bcl-2-associated X protein; Bcl-2, B-cell lymphoma 2; BR, brusatol; CDDP, cisplatin

aforementioned results, the present study aimed to further determine the mechanisms underlying the synergistic antitumor effects of BR and CDDP. Since BR and CDDP cotreatment markedly induced synergistic regulation of apoptosis, the present study focused on the molecular mechanisms underlying apoptosis. In the present study, western blot analysis was used to detect the protein expression levels of procaspase-3, procaspase-9, cytochrome $c$, Bax and Bcl-2. As shown in Fig. 6, the expression levels of procaspase- 3 and procaspase- 9 were markedly decreased following treatment with BR or CDDP alone. Compared with in the monotherapy groups, BR and CDDP cotreatment significantly downregulated the protein expression levels of procaspase- 3 and procaspase- $9(\mathrm{P}<0.05)$ and upregulated the protein expression levels of cytosolic cytochrome $c$ $(\mathrm{P}<0.01)$. The present study also measured the expression levels of Bax and Bcl-2 (Fig. 7). Treatment with CDDP was able to markedly increase the expression levels of $\mathrm{Bax}(\mathrm{P}<0.05)$ and decrease $\mathrm{Bcl}-2$ expression $(\mathrm{P}<0.01)$. In addition, $\mathrm{BR}$ monotherapy significantly decreased the expression levels of Bcl-2 $(\mathrm{P}<0.05)$. However, there was no significant difference between $\mathrm{BR}$ or CDDP monotherapy and cotreatment on the expression levels of Bax and Bcl-2. Furthermore, the Bax/Bcl-2 ratio was also increased $(\mathrm{P}<0.01)$ following monotherapy or cotreatment. These results indicated that BR and CDDP induced cellular apoptosis via a caspase-dependent signaling pathway.

\section{Discussion}

The present study aimed to evaluate the synergistic effects of BR and CDDP on CT-26 CRC cells and to evaluate the possible underlying mechanism. BR is the major active constituent of $B$ javanica, and has been reported to exert potent anti-inflammatory (16), antimalarial (17) and antitumor activities (18-21). CDDP is a chemotherapy drug commonly used in cancer therapy; however, the side effects, including digestive tract reactions, renal toxicity, bone marrow suppression and auditory neurotoxicity, cannot be ignored (25). Furthermore, long-term use of CDDP can induce drug resistance $(26,27)$. Compared with single-drug therapy, it has been reported that combination therapy offers numerous advantages, including several critical molecular targets, lower dose and toxicity, and increased sensitivity (28). Therefore, the present study investigated the synergistic effects of BR and CDDP on CT-26 cells.

The proliferative capacity of tumor cells is deemed vital for the growth and development of tumors (29). The present study demonstrated that a series of concentrations of BR and CDDP dose-dependently suppressed the proliferation and growth of CT-26 cells (Fig. 2). In addition, the results revealed that BR may exhibit a synergistic effect with CDDP on CT-26 cells, with a CI value $<1$.

Apoptosis serves a central role in regulating normal tissue equilibrium, and dysregulation of apoptosis presents a key factor in the growth of cancer (30). Therefore, strategies that target the apoptotic process may inhibit CRC development. Apoptotic cells exhibit characteristics, including cell shrinkage, and chromatin and nuclear condensation $(31,32)$. To determine whether the inhibition of CT-26 cellular proliferation induced by BR and CDDP was associated with apoptosis, morphological alterations were detected by Hoechst 33342 staining following treatment with BR and CDDP for $48 \mathrm{~h}$. The treated 
cells displayed marked apoptotic characteristics, including cell shrinkage, formation of small vesicles, cytoplasmic condensation, pyknotic chromatin and nuclear fragmentation (Fig. 4). The nuclei of CT-26 cells in the cotreatment group appeared to be slightly smaller with brighter fluorescence compared with those of the monotherapy and control groups.

During early apoptosis, phospholipid asymmetry takes place prior to disintegration of the cellular membrane $(33,34)$. Phosphatidylserine (PS) may translocate to the outer layer of the plasma membrane from the inner layer, where it is finally exposed to the external surface of the cell. Therefore, surface exposure of PS is regarded as a sensitive marker for assessing cellular membrane function and apoptosis. Annexin V is a type of calcium-dependent phospholipid-binding protein with a high affinity for PS; its application with PI (a supravital fluorescent dye) is commonly used to detect apoptotic and/or necrotic cells $(34,35)$. To further quantify the apoptotic rate of CT-26 cells following various treatments, cells were stained with Annexin V and PI, and were subjected to flow cytometry. Compared with the percentage of apoptotic cells in the control group $(3.00 \%), \mathrm{BR}, \mathrm{CDDP}$ and cotreatment significantly increased the percentage of apoptotic cells to $13.88,14.21$ and $21.81 \%$, respectively; apoptotic rate was relatively higher in the BR and CDDP cotreatment group.

Activation of caspase cascades is vital for the initiation of apoptosis $(27,36)$. It has been reported that the initiation of apoptosis involves the participation of at least two distinct apoptotic pathways, including the intrinsic mitochondrial apoptotic pathway, which is associated with caspase-9 activation, and the extrinsic apoptotic pathway, which is associated with caspase-3 activation $(37,38)$. To elucidate the molecular mechanism underlying the apoptosis of CT-26 cells induced by BR and CDDP cotreatment, the present study further investigated the possible activation of intrinsic and extrinsic caspase cascades. In the present study, the protein expression levels of procaspase-3 and procaspase- 9 in CT-26 cells treated with BR or CDDP monotherapy, or with a combination of BR and CDDP, were significantly decreased compared with in the untreated cells $(\mathrm{P}<0.05)$, whereas the expression of cytosolic cytochrome $c$ was significantly upregulated $(\mathrm{P}<0.05)$. BR combined with CDDP led to synergistic regulation of the protein expression of initiator and effector caspases in CT-26 cells (Fig. 6). Therefore, it may be suggested that apoptosis of CT-26 cells is induced by BR and CDDP via downregulation of procaspase- 3 and procaspase-9, and upregulation of cytochrome $c$, which may be associated with both intrinsic and extrinsic mitochondrial pathway.

The Bcl-2 family proteins, including Bcl-2 and Bax, also serve an important role in regulation of the mitochondrial apoptotic pathway (39). Released cytochrome $c$ binds with cytosolic apoptosis protease activating factor, and induces the activation of caspase-9 (38). Bcl-2 suppresses the initiation of apoptosis and promotes cell survival by inhibiting the release of cytochrome $c$. Conversely, Bax elicits apoptosis and evoked cell death through its promotion of cytochrome $c$ release from the mitochondria (40). Positive modulation of the Bax/Bcl-2 ratio can lead to decreased mitochondrial membrane potential and the release of cytochrome $c$, thereby contributing to activation of the intrinsic apoptotic pathway. Therefore, the ratio of Bax/Bcl-2 is commonly employed as an important index for the assessment of mitochondria-mediated apoptosis (39).
The present study detected the protein expression levels of proapoptotic Bax, anti-apoptotic Bcl-2 and the Bax/Bcl-2 ratio in CT-26 cells by western blotting. Compared with in the control cells, the expression levels of Bax were significantly enhanced in the treated cells, whereas the protein expression levels of Bcl-2 were markedly decreased (Fig. 7). This observation may result in the release of cytochrome $c$ from the mitochondria, which may further induce apoptosis.

In conclusion, the present study demonstrated that BR could synergistically enhance the antitumor effects of CDDP on CT-26 cells via the intrinsic and extrinsic apoptotic pathways, as indicated by activation of Bax and cytochrome $c$, and negative modulation of procaspase-3, procaspase-9 and Bcl-2. These findings suggested that BR and CDDP cotreatment may be a beneficial option to enhance the antitumor effects of CDDP on the treatment of CRC.

\section{Acknowledgements}

The present study was supported by grants from the Hong Kong, Macao and Taiwan Science and Technology Cooperation Program of China (grant no. 2014DFH30010), the Chinese Medicinal Scientific Research Project of Guangdong Province (grant no. 20161096), the Chinese Medicinal Scientific Research and Technology Research Projects of Guangdong Provincial Hospital of Chinese Medicine and the Key Discipline Construction Projects of Integrative Medicine of High Level University of Guangdong Province (grant no. YN2015QN05), the Science and Technology Planning Project of Guangdong Province (grant no. 2017A050506044), the Natural Science Foundation of Guangdong Province (grant no. 2017A030310124), the Science and Technology Planning Project of Guangzhou of Guangdong Province (grant no. 201704030028), and the China Postdoctoral Science Foundation (grant nos. 2016M600649 and 2017T100622).

\section{Competing interests}

The authors declare that they have no competing interests.

\section{References}

1. Weitz J, Koch M, Debus J, Höhler T, Galle PR and Büchler MW: Colorectal cancer. Lancet 365: 153-165, 2005.

2. Lindblom A, Zhou XL, Liu T, Liljegren A, Skoglund J and Djureinovic T; Swedish Low-Risk Colorectal Cancer Group: Colorectal cancer as a complex disease: Defining at-risk subjects in the general population - a preventive strategy. Expert Rev Anticancer Ther 4: 377-385, 2004.

3. Jemal A, Bray F, Center MM, Ferlay J, Ward E and Forman D: Global cancer statistics. CA Cancer J Clin 61: 69-90, 2011.

4. Torre LA, Bray F, Siegel RL, Ferlay J, Lortet-Tieulent J and Jemal A: Global cancer statistics, 2012. CA Cancer J Clin 65: 87-108, 2015.

5. Zauber AG, Lansdorp-Vogelaar I, Knudsen AB, Wilschut J, van Ballegooijen $M$ and Kuntz KM: Evaluating test strategies for colorectal cancer screening: A decision analysis for the U.S. Preventive Services Task Force. Ann Intern Med 149: 659-669, 2008.

6. Lambert R, Sauvaget C and Sankaranarayanan R: Mass screening for colorectal cancer is not justified in most developing countries. Int J Cancer 125: 253-256, 2009.

7. Xu AG, Yu ZJ, Jiang B, Wang XY, Zhong XH, Liu JH, Lou QY and Gan AH: Colorectal cancer in Guangdong Province of China: A demographic and anatomic survey. World J Gastroenterol 16: 960-965, 2010. 
8. Folkesson J, Martling A and Kodeda K: Current considerations in colorectal cancer surgery. Colorectal Cancer 4: 167-174, 2015.

9. Gustavsson B, Carlsson G, Machover D, Petrelli N, Roth A, Schmoll HJ, Tveit KM and Gibson F: A review of the evolution of systemic chemotherapy in the management of colorectal cancer. Clin Colorectal Cancer 14: 1-10, 2015.

10. Dagoglu N, Mahadevan A, Nedea E, Poylin V and Nagle D: Stereotactic body radiotherapy (SBRT) reirradiation for pelvic recurrence from colorectal cancer. J Surg Oncol 111: 478-482, 2015.

11. Marin JJ, Sanchez de Medina F, Castaño B, Bujanda L, Romero MR, Martinez-Augustin O, Moral-Avila RD and Briz O: Chemoprevention, chemotherapy, and chemoresistance in colorectal cancer. Drug Metab Rev 44: 148-172, 2012.

12. Yin SY, Wei WC, Jian FY and Yang NS: Therapeutic applications of herbal medicines for cancer patients. Evid Based Complement Alternat Med 2013: 302426, 2013.

13. Zhao M, Lau ST, Leung PS, Che CT and Lin ZX: Seven quassinoids from Fructus Bruceae with cytotoxic effects on pancreatic adenocarcinoma cell lines. Phytother Res 25: 1796-1800, 2011.

14. Commission CP (ed): Pharmacopoeia of People's Republic of China. Vol. 1. Chinese Medical Science and Technology Press, Beijing, pp174-175, 2010.

15. Fukamiya N, Okano M, Miyamoto M, Tagahara K and Lee KH: Antitumor agents, 127. Bruceoside $\mathrm{C}$, a new cytotoxic quassinoid glucoside, and related compounds from Brucea javanica. J Nat Prod 55: 468-475, 1992.

16. Turpaev K and Welsh N: Brusatol inhibits the response of cultured beta-cells to pro-inflammatory cytokines in vitro. Biochem Biophys Res Commun 460: 868-872, 2015.

17. Allen D, Toth I, Wright C, Kirby G, Warhurst D and Phillipson J: In vitro antimalarial and cytotoxic activities of semisynthetic derivatives of brusatol. Eur J Med Chem 28: 265-269, 1993.

18. Cuendet M, Gills JJ and Pezzuto JM: Brusatol-induced HL-60 cell differentiation involves NF-kappaB activation. Cancer Lett 206: 43-50, 2004.

19. Hitotsuyanagi Y, Kim IH, Hasuda T, Yamauchi Y and Takeya K A structure-activity relationship study of brusatol, an antitumor quassinoid. Tetrahedron 62: 4262-4271, 2006.

20. Mata-Greenwood E, Cuendet M, Sher D, Gustin D, Stock W and Pezzuto JM: Brusatol-mediated induction of leukemic cell differentiation and $\mathrm{G}(1)$ arrest is associated with downregulation of c-myc. Leukemia 16: 2275-2284, 2002.

21. Ren D, Villeneuve NF, Jiang T, Wu T, Lau A, Toppin HA and Zhang DD: Brusatol enhances the efficacy of chemotherapy by inhibiting the Nrf2-mediated defense mechanism. Proc Natl Acad Sci USA 108: 1433-1438, 2011.

22. Olayanju A, Copple IM, Bryan HK, Edge GT, Sison RL, Wong MW, Lai ZQ, Lin ZX, Dunn K, Sanderson CM, et al: Brusatol provokes a rapid and transient inhibition of Nrf2 signaling and sensitizes mammalian cells to chemical toxicity-implications for therapeutic targeting of Nrf2. Free Radic Biol Med 78: 202-212, 2015.

23. Chou TC and Talalay P: Analysis of combined drug effects: A new look at a very old problem. Trends Pharmacol Sci 4: 450-454, 1983.

24. Ashton JC: Drug combination studies and their synergy quantification using the Chou-Talalay method - letter. Cancer Res 75 2400-2400, 2015.
25. Florea AM and Büsselberg D: Cisplatin as an antitumor drug. Cellular mechanisms of activity, drug resistance and induced side effects. Cancers (Basel) 3: 1351-1371, 2011.

26. Li B, Gao Y, Rankin GO, Rojanasakul Y, Cutler SJ, Tu Y and Chen YC: Chaetoglobosin K induces apoptosis and G2 cell cycle arrest through p53-dependent pathway in cisplatin-resistant ovarian cancer cells. Cancer Lett 356: 418-433, 2015.

27. Galluzzi L, López-Soto A, Kumar S and Kroemer G: Caspases connect cell-death signaling to organismal homeostasis. Immunity 44: 221-231, 2016.

28. Lin Y, Yang X, Lu M, Zheng W, Zhang J, Zhuang H and Hua ZC: Herbal compound triptolide synergistically enhanced antitumor activity of vasostatin120-180. Anticancer Drugs 24: 945-957, 2013.

29. Cao W, Li XQ, Wang X, Fan HT, Zhang XN, Hou Y, Liu SB and Mei QB: A novel polysaccharide, isolated from Angelica sinensis (Oliv.) Diels induces the apoptosis of cervical cancer HeLa cells through an intrinsic apoptotic pathway. Phytomedicine 17: 598-605, 2010

30. Kroemer G and Reed JC: Mitochondrial control of cell death. Nat Med 6: 513-519, 2000.

31. Doonan F and Cotter TG: Morphological assessment of apoptosis Methods 44: 200-204, 2008.

32. Kerr JF, Wyllie AH and Currie AR: Apoptosis: A basic biological phenomenon with wide-ranging implications in tissue kinetics. Br J Cancer 26: 239-257, 1972.

33. Fadok VA1, de Cathelineau A, Daleke DL, Henson PM and Bratton DL: Loss of phospholipid asymmetry and surface exposure of phosphatidylserine is required for phagocytosis of apoptotic cells by macrophages and fibroblasts. J Biol Chem 276: 1071-1077, 2001.

34. Vermes I, Haanen C, Steffens-Nakken $\mathrm{H}$ and Reutelingsperger C: A novel assay for apoptosis. Flow cytometric detection of phosphatidylserine expression on early apoptotic cells using fluorescein labelled Annexin V. J Immunol Methods 184: 39-51, 1995.

35. van Engeland M, Nieland LJ, Ramaekers FC, Schutte B and Reutelingsperger CP: Annexin V-affinity assay: A review on an apoptosis detection system based on phosphatidylserine exposure. Cytometry 31: 1-9, 1998.

36. Budihardjo I, Oliver H,Lutter M,Luo X and Wang X: Biochemical pathways of caspase activation during apoptosis. Annu Rev Cell Dev Biol 15: 269-290, 1999.

37. Ashkenazi A and Dixit VM: Death receptors: Signaling and modulation. Science 281: 1305-1308, 1998.

38. Li P, Nijhawan D, Budihardjo I, Srinivasula SM, Ahmad M, Alnemri ES and Wang X; Li P1: Cytochrome $c$ and dATP-dependent formation of Apaf-1/caspase-9 complex initiates an apoptotic protease cascade. Cell 91: 479-489, 1997.

39. Martinou JC and Youle RJ: Mitochondria in apoptosis: Bcl-2 family members and mitochondrial dynamics. Dev Cell 21: 92-101, 2011.

40. Zhao K, Jin M, Chen Q and Zheng PS: Polysaccharides produced by Enterobacter cloacae induce apoptosis in cervical cancer cells. Int J Biol Macromol 72: 960-964, 2015.

This work is licensed under a Creative Commons Attribution-NonCommercial-NoDerivatives 4.0 International (CC BY-NC-ND 4.0) License. 